\title{
Man komme mir nicht mit so Zeugs!
}

Hans Peter Hartmann ${ }^{a}$, Doris Lehner ${ }^{b}$

a pensionierter Flugkapitän, CRM- und HADmedical-Trainer

b Dr. med., Fachärztin für Anästhesie FMH, HADmedical-Trainerin

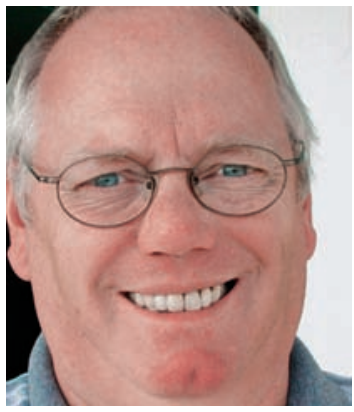

Hans Peter Hartmann

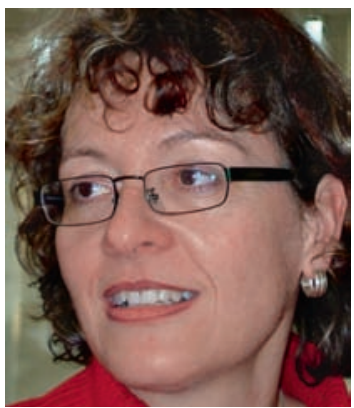

Doris Lehner

Korrespondenz:

Hans Peter Hartmann

Steinacherstrasse 92

CH-8308 Illnau

Tel. 0523472929

info@avi-agency.ch
Immer wieder erfährt man, dass in einem Spital geschah, was nicht geschehen dürfte: Ein Mensch ist nach einer Kaskade von Fehlern zu Schaden gekommen. Ob in einem Spital mehreren Patienten Stick- statt Sauerstoff verabreicht wurde, ob auf der falschen Seite operiert wurde, Röntgenbilder vertauscht oder der falsche Patient in den OP gebracht wurde, meist stecken menschliche Unzulänglichkeiten dahinter. Nach einem einführenden Artikel in der Schweizerischen Ärztezeitung [1] über aktives Fehlermanagement möchten wir in lockerer Folge einige Themen aus dem weiten Bereich «Human Factors» aufgreifen.

\section{Hab' ich das nötig?}

Wie im erwähnten einführenden Artikel beschrieben wurde, werden Flight-Crews seit über 25 Jahren im Bereich «Human Factors» weitergebildet [1]. Offensichtlich ist die Medizin noch nicht soweit. Obwohl einschlägige Kurse für Mediziner angeboten werden, wenngleich mit CIRS bereits ein funktionierendes, anonymes Fehlermeldesystem existiert, kommt eine den Namen verdienende Sicherheits- und Fehlerkultur in der Ärzteschaft nicht so recht in die Hufe, von einem gestreckten Galopp in Richtung wirklicher Einsicht über die Notwendigkeit eines Wandels gar nicht zu reden. Warum? Steht kleinliches Gärtchendenken im Wege? Wird die entsprechende Ausbildung zuwenig gefördert? Sind die Angebote zuwenig bekannt? Es liegt uns fern, die Mediziner belehren zu wollen, doch dürfte manch ein Arzt, manch eine Ärztin oder eine Pflegefachperson beim Weiterlesen Parallelen finden zwischen unseren Berufszweigen, die sich ja auch in vielem gleichen: In beiden Sparten muss das Team oft unter hohem Druck und unter erschwerten Bedingungen funktionieren.

\section{Steile Hierarchien}

Aus Schilderungen in unseren Kursen wissen wir, dass es mit dem Teamgeist nicht in allen OPs und Abteilungen zum besten steht. Leute im OPTeam wurden unflätig angeschnauzt und lächerlich gemacht, und eine OP-Schwester berichtete, wie der Chirurg wutentbrannt ein Instrument zu Boden schmiss, weil sie ihm das falsche Besteck in die Hand gedrückt hatte. Dabei hatte sie ihn einfach falsch verstanden. Er hatte halt etwas in den Bart genuschelt. Plötzlich konnte er deutlich sprechen ...!

Unser Pilot erinnert sich noch gut an die ersten CRM-Seminare in der Fliegerei. Da sassen jeweils um die zwanzig Piloten im Rund, die einen neugierig und erwartungsfroh. Andere, und es

\section{Le «facteur humain» et ses risques}

De plus en plus de gens accusent l'hôpital de fautes et de dommages. A la suite d'un article initiateur sur la gestion active des fautes, nous abordons maintenant un sujet tiré du vaste champ «facteur humain».

Les cours HADmedical nous apprennent que la collaboration n'est pas toujours au beau fixe dans les salles d'opération et les services. La question se pose de savoir si des hiérarchies trop raides y contribuent. Il est arrivé que des personnes soient ridiculisées et «engueulées» de façon ordurière. Une infirmière de salle d'opération a raconté qu'un chirurgien a lancé, de rage, un instrument au sol parce qu'elle lui avait passé un faux instrument. Elle l'avait mal compris car il parlait dans sa barbe. Tout à coup, il $a$ pu s'exprimer clairement ...!

Les expériences faites en aéronautique révèlent que ce sont souvent les «coryphées» qui estiment complètement inutile une formation continue dans le domaine du «facteur humain». Qu'en pensent les autres dans l'avion et la salle d'opération? En tous les cas, ces cours permettent aux pilotes de progresser sur le plan de la sécurité et les «durs à cuire» remarquent avec le temps les avantages procurés par un style de direction véritablement coopératif et ouvert: les collaborateurs sont toujours plus disposés à indiquer les fautes en train de se produire, le courage de s'exprimer augmente en parallèle. 
waren vorwiegend Kapitäne von altem Schrot und Korn, sassen weit zurückgelehnt und mit verschränkten Armen da, die Stirn tief gefurcht, die Mundwinkel spöttisch heruntergezogen, und schon eh der Kurs losging, zischten sie sich hörbar zu: «Müssen wir uns dieses Psychogelaber wirklich anhören? Mich muss jedenfalls niemand lehren, wie man eine Besatzung führt. So einen Kinderkram habe ich nicht nötig!» Worauf die alten Bordingenieure und jungen Kopiloten untereinander bedeutungsvolle Blicke wechselten. War es nicht gerade dieser Kapitän, der auf fast jedem Flug eine Hostess zum Heulen brachte, die Mechaniker zusammenstauchte und den Kopiloten überging, abputzte, blossstellte? Man kennt auch die Sprüche: «Ich befehle jetzt!», «Mein Entscheid wird nicht diskutiert!» Et cetera et cetera. Wäre es möglich, dass Ähnliches auch mal in einem OP verlautbart wurde?

Dann hatte es auch andere Kapitäne in den Kursen. Sie taten nicht so bockbeinig, sie waren gerne bereit, etwas dazuzulernen. Es waren genau diese Kapitäne, die einen solchen Kurs wirklich «nicht nötig» gehabt hätten, wie die Kopiloten fanden.

Über die Jahre hinweg fand tatsächlich ein Kulturwandel statt. Obwohl natürlich der Kapitän nach wie vor die volle Verantwortung trug, wurde die Pyramide doch flacher. Sicher war es auch der Zeitgeist, der einen Wandel brachte, doch führte das gesetzlich vorgeschriebene Training manch Harthölzernem vor Augen, dass er offenbar der einzige war, der im Schritte ging. Manch einer wurde im Verlauf des Tages etwas ruhiger und nachdenklicher und hörte den Diskussionen aufmerksamer zu. Nicht dass sie dann gleich geläutert von dannen gegangen wären. Doch zeigte sich bei der Arbeit im Cockpit immer öfter, dass die Mitarbeiter ihre Meinung kundtun konnten und auch angehört wurden. Eine witzige Episode illustriert die Entwicklung punktgenau: Während einer Flugplanung legte der Dispatcher das Streckenwetter vor, Kapitän und Kopilot beugten sich über die Unterlagen. «Was meinst du zu dem Wetter?» wandte sich der Chef an den Kopi. Dieser äusserte seine Meinung. «Aha», antwortete der Kapitän und fügte, zum Dispatcher gewandt, bei: «Wir müssen halt neuerdings auch noch die Kopiloten um ihre Meinung fragen.» Alle drei brachen in Gelächter aus, und alle wussten, dass sich da wieder ein «Urgestein der Aviatik» um den Zehntel eines Grades gedreht hatte, ohne dass die ganze Autorität zusammengebrochen wäre. Darum konnte der Kopilot auch mit einem Grinsen antworten: «Stimmt!»

\section{Wo bleibt die Autorität?}

Der Widerstand gegen eine Teamarbeit, die den Namen auch verdient, manifestierte sich häufig in der Sorge, die Autorität des Chefs könnte ins Wanken geraten. Doch bitte: Davon, dass ich bei jeder Gelegenheit das Namensschild mit dem Titel poliere und unablässig darauf hinweise, dass ich der Chef bin, wird meine Autorität auch nicht besser. Wenn ich in leitender Funktion laufend den Grossen Zampano spielen muss, stehe ich bald einmal «mit den Rollerblades im Kies»um des Zürcher Kabarettisten Blues Max köstlichen Spruch auszuborgen.

Anders die Chefs, die im OP oder im Cockpit innert Minuten einen Teamgeist $\mathrm{zu}$ schaffen wussten, dass es eine Freude war, mitzudenken, sich zu äussern, Mitverantwortung zu tragen. Klar wurde man auch mal korrigiert, und selbstverständlich hörte man auch in ihrer Stimme gelegentlich jene keinen Verzug duldende Bestimmtheit, wenn etwas zu dringendem, verzugslosem Handeln zwang.

\section{Wer hält den Fuss dazwischen?}

Dieser kooperative, teamorientierte Führungsstil bringt nur Vorteile: Die Mitarbeiter fühlen sich ernst genommen, freuen sich, wesentlich zum Erfolg beigetragen zu haben, sie fühlen sich wohl im Team. Besonders dann, wenn sie Anerkennung für ihr Engagement finden, sich akzeptiert und gefördert fühlen und nach der Arbeit nach Hause gehen können im guten Gefühl, aktiv und mitverantwortlich zum guten Gelingen beigetragen zu haben.

Eine solche Stimmung im Team fördert auch den Mut, Bedenken zu äussern, auf Fehler hinzuweisen, den Fuss dazwischenzuhalten, wenn etwas aus dem Ruder zu laufen droht. Besonders dann, wenn die Mitarbeiter erleben, dass sie auch dann noch für ihre Initiative gelobt werden, wenn sie halt einmal daneben gelegen haben.

\section{Nobody is perfect}

Nur die allergrössten Hornochsen halten sich für unfehlbar. Alle anderen wissen, wie schnell ihnen ein Fehler unterlaufen kann. Sich dann auf ein Team stützen zu können, das einem rechtzeitig in den Arm fällt, schafft Übersicht und Sicherheit.

In einem der HADmedical-Kurse erzählte eine Operationsschwester von einer Herzoperation und vom Moment, als der Operateur gerade dabei war, einen fatalen Fehler zu begehen. Sie erzählte, was in ihr in diesem Moment vorging: 
Erst ungläubiges Staunen, doch blitzschnell erkannte sie, dass sie ihn warnen muss, weil alle anderen gerade auf den Monitor schauten. Den Warnruf hatte sie schon auf der Zunge, doch für einen winzig kleinen Moment hielt sie die etwas zurück, was man einen Flashback nennen könnte. In Blitzesschnelle schoss ihr durch den Kopf, wie das damals war,

- als sie sich kritisch über den Zustand eines Patienten geäussert hatte. Damals hatte ihr der Oberarzt kühl geantwortet: «Davon verstehen Sie nichts»;

- als sie auf einen offensichtlich im Bauch liegengebliebenen Tupfer hinwies und der Chef sie mit herablassendem Unterton anschnauzte: «Ich habe ihn schon gesehen.» Und jeder an dem Tisch wusste: Nein, hatte er nicht;

- als der Anästhesist auf die sachliche und fachlich gerechtfertigte Frage, wie lange die Chirurgen noch für ihre Arbeit bräuchten, zur Antwort erhielt: «So lange, bis wir fertig sind.» Prima! Damit konnte er viel anfangen!

Doch nun war die Situation anders. Mit diesem Chirurgen hatte sie andere Erfahrungen gemacht, und so fasste sie ihn unwillkürlich am Arm und rief: «Halt!»

«Und», fragten wir, «wie ging das weiter?» Sie erzählte, er habe zuerst dagestanden wie vom Donner gerührt, dann habe er sie angesehen und gesagt: «Danke! Du hast mich gerade vor einem Super-GAU gerettet.»

\section{Die allerdümmste Antwort}

Nun müsste man ja eigentlich meinen, diese Reaktion sei völlig normal. Ist sie das? Wie oft bedanken wir uns bei jemandem, der mitdenken und uns vor Unheil bewahren wollte, statt mit einem Dankeschön mit Sätzen wie:

- «Ich hab’ es schon gesehen.»

- «Das weiss ich selber.»

- «Ich hätte/wollte ja gerade ...»
Es sind die allerdümmsten Sätze, die man in einem Cockpit, Operationssaal, Auto oder Büro sagen kann. Wir können sie getrost aus dem Repertoire streichen. Und doch liegen sie uns allzuoft auf der Zunge. Auch den Autoren dieses Artikels, wie mit einem Grinsen zu vermelden ist.

\section{Menschlich eben!}

Man sieht, wie schnell Menschliches ins Spiel kommt. Niemand ist gefeit davor, über die uns allen innewohnenden Unzulänglichkeiten zu stolpern. Es geht darum, wie wir ticken, agieren, reagieren, wahrnehmen, Entscheidungen treffen, kommunizieren, führen. Dies alles kommt ja ins Spiel, und deshalb lohnt es sich, mehr über den Umgang mit menschlichen Faktoren zu erfahren und Erkenntnisse zu gewinnen.

Zweifellos ist unser medizinisches Personal im fachlichen Bereich bestens geschult. Doch wie steht es mit der Selektion und Weiterbildung des medizinischen Personals im Umgang mit menschlichen Faktoren? Die Erfahrung zeigt, dass die Bereitschaft zur Weiterbildung beim Pflegefachpersonal, bei Anästhesistinnen und Anästhesisten unvergleichbar grösser ist als beim Rest der Ärzteschaft. Doch ändert sich das ja vielleicht auch mit der öffentlichen Diskussion zur Frage der Patientensicherheit und zu den Haftungsfragen. Ein erster, erfreulicher Schritt ist damit getan, dass die FMH für den Besuch der HADmedical-Seminare Credit Points vergibt.

Vielleicht führt das ja dazu, dass sich der eine oder die andere auf die Homepage von HADmedical unter www.hadmedical.ch einklickt, um sich zu informieren, ob es da nicht etwas zu vertiefen gäbe. Zum Wohle der Patienten und der eigenen Entdeckungslust zuliebe. Schön wäre es!

\footnotetext{
Literatur

1 Brändle B, Scheidegger D. Aktives Fehlermanagement durch eine teamorientierte Arbeitskultur. Schweiz Ärztezeitung. 2007;88(8):325-7.
} 